Linguistique, littérature, didactique

\title{
Façons de lire, façons de faire
}

Jean-Marie Privat et Marie-Christine Vinson

\section{(2) OpenEdition \\ Journals}

Édition électronique

URL : http://journals.openedition.org/pratiques/1160

DOI : 10.4000/pratiques. 1160

ISSN : 2425-2042

\section{Éditeur}

Centre de recherche sur les médiations (CREM)

\section{Édition imprimée}

Date de publication : 15 juin 2008

Pagination : 199-216

\section{Référence électronique}

Jean-Marie Privat et Marie-Christine Vinson, «Façons de lire, façons de faire », Pratiques [En ligne], 137-138 | 2008, mis en ligne le 15 juin 2008, consulté le 19 avril 2019. URL : http:// journals.openedition.org/pratiques/1160; DOI : 10.4000/pratiques.1160 


\title{
Façons de lire, façons de faire
}

\author{
Jean-Marie Privat, \\ Marie-Christine Vinson
}

Université Paul Verlaine - Metz, CELTED, EA 3474

Dans la maîtrise scolaire des textes, selon le niveau des opérations langagières sollicitées, il apparaît que dominent encore trop souvent les modèles les plus formels de la linguistique structurale et cognitive (repérage des connecteurs, analyse du jeu des rimes, etc.) ou les méthodes, si peu «actives » en fait, inspirées des pédagogies académiques de la communication (fiches de lecture, lecture cursive, etc.)

On s'interroge par contre assez peu, semble-t-il, sur la construction de savoirs-faire «bricolés » en fait par tout lecteur confirmé en ses façons de s'approprier concrètement un document écrit. Ces techniques et tactiques de lecteurs présentent en effet des aspects très concrets et très ordinaires qui sont autant d'actes pratiques de capitalisation de ses lectures : savoir, par exemple, à moindre coût suspendre et reprendre une lecture, mener une relecture qui ne se borne pas à se répéter, annoter son parcours dans son livre.

En confondant impératif culturel (il importe de lire) et travail d'outillage de sa lecture, on risque de brûler les étapes car on intellectualise trop rapidement les habiletés langagières requises pour «traiter » des textes longs et/ou difficiles. $\mathrm{Au}$ risque de renforcer, sans le vouloir, l'insécurité culturelle et linguistique, normale par ailleurs chez de jeunes apprenants, face à des ensembles textuels riches ou nouveaux. La compréhension réelle et l'interprétation personnelle ne peuvent qu'en souffrir.

Aussi l'enjeu du travail qui va être présenté est bien la mise en œuvre explicite de quelques gestes fondamentaux de la médiation textuelle ${ }^{(1)}$.

Dans le cadre d'un travail en projet ${ }^{(2)}$, on propose à une classe de sixième d'u-

(1) Pour un développement plus approfondi de la problématique des médiations textuelles voir J.-M Privat et alii, "Vers une didactisation des médiations textuelles », Cahiers du Français Contemporain, $\mathrm{n}^{\circ} 7$, Pratiques de lecture et cheminements du sens, coordonné par Michel Dabène, ENS Editions, Lyon, 2001,pp. 161-177.

(2) Nous aimerions souligner ici combien notre réflexion est redevable des travaux de Jean- 
ne vingtaine d'élèves de s'organiser en comité de lecture pour choisir deux livres auxquels elle décernera un « Grand Prix » et un «Prix Spécial ».

Chaque élève se trouve ainsi engagé dans une activité de lecture qui va lui demander de lire au moins quatre livre, sur une durée de cinq semaines. Au cours de cette activité, le jeune lecteur sera contraint de suspendre et reprendre sa lecture ; il lui faudra baliser le texte lu par des annotations qu'il inscrira sur une feuille ; il sera amené à repérer les pages susceptibles d'être sautées pour progresser plus rapidement; il devra aussi relire des passages plus ou moins longs pour étayer son jugement lors du choix final.

Ces différentes situations devraient permettre aux élèves de mettre en œuvre, développer, objectiver et rationaliser des opérations lectorales à la croisée de la maîtrise pratique et symbolique de l'appropriation des textes. C'est ce que nous entendons par construction raisonnée de médiations textuelles ${ }^{(3)}$.

\section{Un lecteur, apprenti médiateur textuel (Lire le premier livre)}

\subsection{Construire un corpus}

La première séance de travail sert à élaborer le corpus des livres mis en lecture. Il est proposé aux élèves d'explorer le fonds des séries de romans stockées au CDI du collège.

Pourquoi utiliser des séries d'ouvrages de fiction dans un tel apprentissage ? C'est disposer facilement d'un nombre de livres conséquents sans avoir à y consacrer un budget important. C'est surtout décider de rompre avec un usage routinier du lire à l'école : un même ouvrage pour tous à étudier dans un même temps.

Une trentaine de livres différents sont ainsi répertoriés et apportés en classe, uniquement ceux destinés par l'institution aux $6^{\mathrm{e}} / 5^{\mathrm{e}}$. Mais de l'avis unanime de la classe, trente livres représentent une quantité de textes à lire difficile à envisager ! Il faut donc opérer une première sélection de façon à réduire significativement le nombre d'ouvrages. Trois groupes de six à sept élèves sont constitués; ils ont en charge une dizaine de livres. Ils doivent en sélectionner trois ou quatre après un premier feuilletage et une première discussion. Rapidement on convient que pour réaliser au mieux la sélection, il faut dégager des critères pertinents c'est-à-dire adapter au projet. Ainsi les élèves se mettent d'accord pour éliminer :

les livres trop longs

les livres trop faciles ou trop difficiles

les livres connus

les livres déjà lus

Dix livres sont retenus à partir desquels les activités de lecture proprement dites vont pouvoir être lancées ${ }^{(4)}$.

François Halté sur le sujet et combien le fameux numéro "Travailler en projet » (Pratiques, 1982,36) a profondément changé notre façon de penser les apprentissages scolaires.

(3) Sur des dispositifs proches mais où l'accent est mis sur les dynamiques de l'acculturation lettrée, voir par exemple M. Burgos et J.-M. Privat, «Le Goncourt des lycéens : vers une sociabilité littéraire ? ", Lire en France aujourd'hui, sous la direction de M. Poulain, Paris, Editions du Cercle de la Librairie, Collection Bibliothèques, 1993, pp. 163-181.

(4) Voir fiche $n^{\circ} 1$ en annexes. 


\subsection{Inventorier «Mes » façons de lire}

Un nouveau dispositif de travail est mis en place pour organiser la lecture du premier livre (séance 2).

Cette fois les élèves se répartissent en cinq groupes de quatre. Chaque groupe reçoit deux livres différents, en deux exemplaires. Ainsi, par binome, les élèves lisent le même livre. Est également distribuée une fiche intitulée "Mes conseils de lecteurs ${ }^{(5)}$. Elle est remplie au cours, mais surtout après la lecture. Cette fiche, élaborée par l'enseignant, joue le rôle de médiation. Elle doit faire bénéficier le second lecteur de l'expérience liseuse du premier lecteur. Ce premier lecteur est ainsi placé d'emblée en situation de médiateur textuel. Pour le guider dans ce travail, la fiche propose plusieurs pistes.

Il s'agit d'abord de quantifier le temps de la lecture - évaluer concrètement l'investissement temporel que nécessite la lecture d'un livre - pour dégager les grandes unités chronotopiques qui scandent l'avancée dans un texte et qui sont comme les traces laissées par le lecteur lui-même.

Ensuite, interroger chacun sur son cheminement dans les textes pour faire prendre la mesure de la diversité des parcours possibles. En formulant des choix pour alléger la tâche d'un pair-lecteur et préserver son intérêt à la lecture, les élèves retravaillent les modèles les plus scolaires de la lecture exhaustive et linéaire. Ils apprennent à sauter des pages, à rompre le continuum du texte, mais également à renouer les fils du sens pour maintenir la cohérence d'ensemble. Pour ce faire, chaque saut dans le texte est accompagné d'un commentaire « Lis mes raccourcis », adressé au lecteur suivant. Aux accélérations liseuses peuvent répondre des ralentissements. Il s'agit, par exemple, de cerner des passages qui demandent une attention soutenue et un effort particulier, compte tenu de leur importance dans l'économie locale ou générale du roman.

C'est donc tout un travail sur le rapport au temps qui est en jeu dans les deux premières questions posées. L'objectif est de faire découvrir aux jeunes lecteurs la liberté dont ils disposent pour organiser leur temps :

« Le livre est quelque chose qui prend du temps : on peut en lire un bout aujourd'hui, un autre demain, en reporter la lecture à plus tard, ralentir sa lecture ou l'accélérer, etc. Avec le livre, nous sommes dans une culture du stock : on gère le livre comme on l'entend. Ce qui est impossible dans une culture de flux : avec la télé, avec la radio, on peut certes décider de tout regarder ou de n'en regarder qu'une partie, mais on n'a pas la maîtrise du déroulement du temps, on ne peut réagir qu'à un temps qui nous est imposé, très peu de gens utilisent la faculté du ralenti ou de l'arrêt sur image. Et toute interruption de l'attention laisse échapper un contenu qui continue à se dérouler hors de l'intervention du récepteur. » ${ }^{(6)}$

Les activités de domestication textuelle dans lesquelles les élèves sont engagées concernent aussi la compréhension du texte. Dans cette perspective, il convient de solliciter des rapprochements et d'élaborer des explicitations qui vont faciliter la première lecture et surtout les suivantes. La reformulation d'un passage dans un langage personnel ou le recours à la paraphrase sont autant de stratégies pour rassurer les jeunes lecteurs sur leurs pouvoirs face aux livres. Enfin,

(5) Voir fiche $n^{\circ} 2$ en annexes.

(6) Jean-François Barbier-Bouvet, "Internet, lecture et culture de flux », Esprit, Décembre 2001, pp. 20-33. 
insérer des post-it entre les pages (trois post-it « super » et trois post-it «bof») inscrit la présence du lecteur dans le texte lu. Il interagit avec le livre en donnant son avis, en rédigeant des remarques personnelles. Il interpelle aussi le lecteur suivant et amorce le dialogue qui affilie à la communauté des pairs-lecteurs.

Si l'on considère la lecture comme une pratique dialogale que des interactions outillées inscrivent dans des espaces discursifs, il est nécessaire d'objectiver délibérément l'usage de ces procédures et même de rationaliser leur apprentissage. C'est pourquoi les items proposés amènent les élèves à prendre conscience des différentes tâches qu'ils accomplissent ou doivent accomplir en tant que lecteur pour s'approprier le texte. L'enjeu de cette distanciation métacognitive est important car cette posture tend à faciliter le contrôle de ses propres opérations de traitement cognitif des données discursives ${ }^{(7)}$. Parce qu'ils s'adressent à des pairs qu'ils doivent aider, les jeunes lecteurs non seulement s'observent, mais encore affinent leurs pratiques à travers les conseils qu'ils rédigent.

Lors de la troisième séance, la lecture du premier livre se poursuit et s'achève pour la majorité des groupes (lecture en classe et à la maison). Il s'agit maintenant pour chacun de renseigner la fiche $n^{\circ} 2$. Les élèves sollicitent éventuellement l'enseignant pour les aider à répondre aux questions. Aurélie, après réflexion, évalue à 3 heures 30 sa lecture du Bon Gros Géant de R. Dahl ; Mathieu met un post-it pour attirer l'attention sur le séjour en prison du héros du Faucon déniché, un passage qu'il trouve excellent. Guillaume suggère de sauter la description du château dans Le sorcier aux loups de P. Thiès alors que Morgane signale deux pages « un peu difficiles » à lire attentivement, le raisonnement d'Emilien dans Baby-sitter blues de M.-A. Murail.

Les élèves ne sont pas habitués à ces questions qui valorisent la façon dont le lecteur s'y prend pour lire le texte plutôt que le texte lui-même. Alors comment clarifier l'enjeu d'un travail qui nécessite la mise à jour de savoirs-faire pratiques habituellement abandonnés à la clandestinité, rarement conscients, souvent invisibles mais de fait incontournables?

\subsection{Confronter « Nos » façons de lire}

Les élèves qui ont lu le même livre échangent leur fiche (séance 4). Accords et désaccords apparaissent. Mais, également une compréhension, parfois insuffisante, des enjeux de certaines rubriques. Il faut revenir, par exemple, sur l'intérêt d'accélérer (en sautant des pages) ou de ralentir sa lecture. En effet lire en « piqué » (survoler la page et prélever un élément notable), en " rase-mottes » (lire minutieusement, à même chacun des mots) fait expérimenter aux élèves une posture fondatrice de l'activité liseuse. «Le lecteur est pluriel » dit Roland Barthes et « pour un texte il y a une multitude de lecteurs : non pas seulement des individus différents, mais aussi dans chaque corps des rythmes différents d'intelligence, selon le jour, selon la page ${ }^{(8)}$.

(7) Les travaux que nous menons sur les gestes de lecture et la maîtrise pratique des textes peuvent être rapprochés de ceux déjà engagés sur l'écriture extrascolaire. Et nous pourrions reformuler ainsi la problématique générale : dans quelle mesure la connaissance de ces pratiques peut-elle intéresser la didactique de la lecture? Voir M.-C Penloup, «Questions scolaires à l'écriture extrascolaire », Pratiques, n²115-116, décembre 2000, pp. 1-9.

Roland Barthes, Sollers écrivain, Seuil, 1979, p. 75. 
Pour rendre plus explicites ces divers régimes de lecture, il est proposé à la classe de penser à une pratique qu'elle connaît bien, celle des rediffusions des matches de football à la télévision. Un match, fût-il, un match de Coupe du Monde, sera rarement retransmis lors du bilan de fin de semaine dans son intégralité : les temps morts de la partie seront sautés au profit des moments forts du jeu. Inversement, les belles actions seront montrées au ralenti pour que le spectateur puisse apprécier la complexité des stratégies et la finesse des passes.

Après confrontation des travaux et discussion entre les élèves qui ont lu le même livre, une troisième fiche est donnée par l'enseignant : "Nos conseils de lecteurs ${ }^{(9)}$. Si les items retenus sont identiques à ceux de la fiche précédente (fiche $n^{\circ} 2$ ), une synthèse négociée des remarques des deux lecteurs est nécessaire pour la remplir. Aurélie et Pauline se mettent d'accord sur les unités de texte susceptibles d'être sautées : le passage où le Bon Gros Géant parle de ses oreilles et le passage où il prend son petit déjeuner avec la reine. Par contre elles ont plus de mal à rédiger des « raccourcis » satisfaisants : elles s'opposent sur des problèmes de contenu et de longueur de la reformulation. Arnaud et Christophe tombent rapidement d'accord sur le fait que La Cour aux étoiles de E. Brisou-Pelen leur rappelle le film Les Visiteurs.

Le temps de la lecture n'est pas toujours facile à déterminer et certains élèves se lancent dans des calculs de moyennes pour trouver un point d'entente. La coopération lectorale qui s'instaure suscite donc des échanges de pratiques nombreux et variés.

\section{Un lecteur outillé et critique (lire le deuxième et troisième livre)}

Une fois la fiche "Nos conseils de lecteurs » remplie en deux exemplaires, elle est glissée dans les ouvrages correspondants. Les livres ainsi "équipés » sont échangés à l'intérieur du groupe. Ce n'est plus un livre nu qui arrive entre les mains du lecteur mais un livre accompagné des signes laissés par les lecteurs précédents. Les élèves d'ailleurs ne s'y trompent pas : dés que l'ouvrage est en leur possession, ils se précipitent sur les post-it. Ce simple dispositif de communication favorise, sur un mode pratique et ludique, la socialisation du texte en prenant en compte les appréciations d'un pair.

Au cours de cette cinquième séance, les élèves se lancent également dans la lecture de leur deuxième roman. Un grand calme règne dans la classe qui s'investit totalement dans 1'activité. Quelques lecteurs subvocalisent. Deux garçons ont du mal à dépasser les vingt minutes de lecture silencieuse ; ils essaient, sans succès, de détourner l'attention de leurs camarades proches.

La lecture du deuxième livre est terminée à la séance suivante (séance 6). À ce moment du travail, chaque groupe de quatre élèves a lu deux livres. Les cinq groupes que forme la classe se sont donc appropriés les dix livres du corpus initial. De plus, les élèves ont rempli deux fiches, "Mes conseils de lecteur 》 rédigée individuellement et "Nos conseils de lecteurs » rédigée avec un camarade. Cette dernière fiche leur a également servi d'aide à la lecture. Tout l'intérêt du travail réalisé réside dans l'inventaire ouvert des possibilités d'interventions du lecteur dans le texte que les situations proposées permettent de dresser.

(9) Voir fiche $n^{\circ} 3$ en annexes. 


\subsection{Dégager des critères de lecteurs}

En liaison avec les expériences liseuses vécues par les élèves, il leur est demandé, lors de cette sixième séance, de choisir le livre qu'ils préfèrent parmi les deux qu'ils viennent de lire.

Pour étayer ce choix, une liste de critères est rédigée au tableau. Les propositions, nombreuses, sont notées dans l'ordre où elles sont données. Quelques exemples permettront de se rendre compte de leur diversité : humour, vocabulaire proche du lecteur, nombre de pages, genre, succès du livre, collection, rapport au réel, qualité du titre... Ce foisonnement - le tableau est couvert d'inscriptions - justifie aux yeux des élèves l'intérêt d'une réflexion commune. Parmi les vingt-six critères dégagés, cinq sont privilégiés : découverte d'un auteur nouveau, intérêt maintenu tout au long du roman, intérêt du thème dominant, texte compréhensible, typographie claire et lisible.

Muni de cette liste, chaque groupe sélectionne son roman. Il reste alors cinq livres en course (un par groupe) : Le Bon Gros Géant de R. Dahl, Le Sorcier aux loups de P. Thiès, La Rédac de R. Reberg, Baby-Sitter Blues de M.-A. Murail et Double Meurtre à l'Abbaye de J. Mirande.

Les livres sélectionnés sont dotés d'un nouvel outil de guidage, la fiche ${ }^{\circ} 4$ (que nous commenterons en 2.2) qui reprend tout les modifiant sensiblement, les fiches précédentes. Une fois remplie, cette fiche est glissée dans le livre et chaque roman change de groupe (séance 7). Un temps est alors donné pour lancer la lecture silencieuse du troisième livre.

Quand la classe se retrouve (séance 8), la lecture de ce troisième livre est achevée. Afin que le projet ne s'enlise pas dans la durée, il est proposé une nouvelle sélection. Chaque groupe va juger et noter le troisième livre qu'il vient de lire par rapport au précédent : lequel préfère-t-il ? Pour réaliser ce choix, les élèves utilisent une grille de critères ${ }^{(10)}$ qui est une reprise des critères notés au tableau lors de la séance 7. Il ne s'agit pas d'une reprise à l'identique, mais d'un véritable travail de structuration qui demande de regrouper, de dégager des rubriques. Avec l'aide de l'enseignant, la classe propose lisibilité, intérêt, classification, objet-livre... Ces entrées constituent pour chacun des éléments incontournables à convoquer dans le choix d'un livre. Pour rendre la grille plus performante, on souligne, par rubrique, un seul critère désigné comme majeur.

Ainsi Morgane qui vient de terminer avec son groupe la lecture de La Rédac doit comparer ce roman à Baby-Sitter Blues qu'elle a lu précédemment. Elle doit, un peu comme au patinage artistique, donner une note allant de 1 (note faible) à 5 (bonne note) à chacun des critères retenus. Le texte de La Rédac est-il plus ou moins compréhensible que Baby-Sitter Blues? L'intérêt est-il maintenu tout au long de façon plus satisfaisante dans La Rédac que dans Baby-Sitter Blues ? Est-ce que E. Reberg a un style (une façon d'écrire) plus original que celui de M.-A. Murail ? Est-ce que la mise en page, le choix des caractères typographiques sont plus «confortables » pour le lecteur dans Baby-Sitter Blues que dans La Rédac?

Le Bon Gros Géant de R. Dahl, La Rédac d'E. Reberg, Le Sorcier aux loups de P. Thiés obtiennent les plus gros scores. Ils restent en compétition. Sont éliminés Baby-Sitter Blues de M.-A. Murail et Meurtre à l'Abbaye de J. Mirande.

(10) Voir fiche $n^{\circ} 5$ en annexes. 


\section{2. «Bricoler un outil» de lecteur}

Revenons sur 1'élaboration de la fiche ${ }^{\circ}{ }^{4}$. Quand débute la séance 7, chaque groupe se retrouve avec le livre qu'il a choisi distribué en quatre exemplaires, un pour chaque élève. Avant que ce roman ne change de mains et de lecteur, un nouvel outil facilitant son appropriation est proposé : la fiche "Nos nouveaux conseils de lecteurs " ${ }^{(11)}$. Elle s'inspire très fortement des fiches antérieures, mais pour la réaliser, l'enseignant tient compte des remarques critiques faites par les usagers. En effet les avis sur l'efficacité de la fiche "Nos conseils de lecteurs" ont été assez contrastés. Certains ne les ont pas vraiment utilisées car elles n'étaient pas remplies avec suffisamment de précision; ils le regrettent. D'autres ne sont pas toujours d'accord avec la pertinence des informations données; ils le font savoir. D'autres encore ont suivi les indications avec profit.

Ainsi, par exemple, la rubrique post-it est placée en première position : tout le monde convient que c'est une excellente accroche pour entrer en lecture. Curiosité vis-à-vis d'autrui, mais aussi envie de réagir, de confronter ses propres réactions. La formulation des questions peut elle-même être modifiée : il ne s'agit plus de déterminer "les grandes étapes du texte à lire » pour mieux organiser son temps de lecture, mais de dégager "les grands blocs de texte qu'il vaut mieux lire sans s'arrêter. " En effet, le mot étapes a été jugé peu satisfaisant car il évoquait pour plusieurs élèves les étapes du récit (au sens de l'analyse structurale). Or, les unités du lecteur (les morceaux de texte lus) ne coïncident pas obligatoirement avec les unités narratologiques (les éléments du récit). Le mot bloc lui-même, est choisi, si l'on peut dire, faute de mieux : le lexique officiel du lecteur manque de mots !

Des explicitations sont également apportées. Ainsi «les passages que nous te conseillons de lire avec plus d'attention » s'augmente d'une parenthèse justificative où le lecteur s'autorise à commenter plus précisément le texte, en fait à prendre position. L'item " pour que tu avances plus vite que nous, nous t'expliquons... » se subdivise en deux sous-entrées qui délimitent plus finement les niveaux d'interventions du lecteur : " quelques mots importants que nous avons trouvés difficiles », "ce passage du livre qui n'est pas très clair à première lecture et sur lequel on a intérêt à réfléchir. » Toutes ces modifications sont le résultat de l'expérience réfléchie des lectures précédentes.

Par groupe, les élèves remplissent la fiche «Nos nouveaux conseils de lecteurs » Ils ont tous lu le livre retenu; il leur est donc possible de confronter leurs avis, de négocier, de proposer des réponses développées qui rendent compte de réaction opposées. Caroline, Déborah, Lindsay et Elodie conseillent de lire avec plus d'attention un passage de La Rédac parce qu' " on s'imagine vivre avec Eric (le héros). Pour échapper à l'écriture de sa rédac, il invente un kidnapping et s'enfuit chez un copain. On trouve ce passage amusant parce qu' on n'oserait pas faire la même chose ! » Alexandre, Daniel, Mathieu et Guillaume invitent à réfléchir dans Le Sorcier aux loups sur le passage où "Saint-Roch est considéré comme une bête. L'auteur le décrit souvent comme s'il était lui-même un loup (yeux jaunes, force sauvage...) Cela t'aidera à comprendre la relation entre Saint-Roch et les loups. " Morgane, Souad, Laetitia et Coralie trouvent le mot «prédestiné » difficile et proposent l'explication suivante : Comme il s'ap-

(11) Voir fiche $n^{\circ} 4$ en annexes. 
pelle Richard, il est prédestiné à être riche plus tard. » Pauline, Aurélie, Bastien et Flavien ne parviennent pas à se mettre d'accord sur la durée de la lecture du Bon Gros Géant, ils inscrivent sur la fiche « $3 \mathrm{~h}$ ou $4 \mathrm{~h}$, cela dépend si tu es un lecteur rapide ou pas. "

Mieux renseignée, la fiche a pleinement joué son rôle de médiations textuelles, elle a suscité une curiosité amusée à la façon de lire des autres et une attention intéressée à ses propres «bricolages » de lecteur. Elle a favorisé les interactions et donc incité à des modifications plus ou moins grandes de ses pratiques.

Une fois cette fiche remplie, un exemplaire est glissé dans chacun des livres qui sont échangés entre les groupes.

\section{Un lecteur « re-lecteur" (Lire le quatrième livre)}

Pour la lecture du quatrième texte, une nouvelle organisation de la classe est mise en place (séance 9). Trois groupes sont constitués en relation avec les trois romans sélectionnés : Le Bon Gros Géant, La Rédac, Le Sorcier aux loups. Chaque groupe de six ou sept élèves lit le même texte distribué en autant d'exemplaires que nécessaire. Pour accompagner cette dernière lecture, les élèves disposent d'une fiche intitulée "Lire-Annoter-Relire » ${ }^{(12)}$ Cette fiche invite le lecteur à prendre position par rapport à l'histoire qu'il est en train de lire, elle le guide dans sa relecture pour qu'il puisse étayer son jugement final lors de la remise des prix. Les élèves sont donc placés dans une situation qui propose une finalité (pourquoi ?) et un dispositif d'accompagnement (comment ?) à la relecture.

Certes, l'école enjoint de relire, mais les stratégies d'ensemble ou les tactiques locales sont rarement explicitées. Ce type d'injonction dissimule les opérations qui orientent la relecture. Comment alors affiner l'interprétation du texte en pratiquant une relecture efficace?

\subsection{Baliser son parcours de relecteur}

Il s'agit tout d'abord de préparer le travail de relecture. En fonction du projet « donner son avis de lecteur pour décerner un prix », chacun doit réagir personnellement au fil de sa lecture. Cette valorisation des réactions liseuses et des partis-pris écrits se traduit dans la pratique par un protocole simple. On convient d'entourer au crayon à papier le numéro de la page quand :

- « j'aimerais ressembler au personnage »

- « je suis d'accord avec ce qu'il fait»

- «je le trouve amusant, mystérieux, intelligent... »

- « je trouve que l'histoire est bien trouvée»

- « je trouve que la page est intéressante»

Il faut barrer au crayon le numéro de la page quand :

- « je n'aimerais pas ressembler au personnage»

- « je ne suis pas d'accord avec ce qu'il fait»

- « je ne le trouve pas intéressant»

- « je trouve que l'histoire est banale, ennuyeuse »

- « je trouve la page trop compliquée »

(12) Voir la fiche $n^{\circ} 6$ en annexes. 
Quand la lecture du quatrième livre est terminée, les élèves se retrouvent en groupes pour échanger leurs impressions sur les pages sélectionnées. Ils discutent, comparent, acquiescent ou contestent les choix faits par les uns et les autres (séance 10). Cet échange libre et plutôt informel va être suivi par un travail plus précis et plus construit.

\subsection{Relire}

Dans L'art de lire (1911) ${ }^{(13)}$, le critique littéraire Emile Faguet intitule l'avant dernier chapitre « Relire». Placé juste avant l'épilogue, ce chapitre vient clore, comme un point d'orgue, la réflexion. Quatre raisons à l'acte de relire sont clairement énoncées. Tout d'abord on relit pour mieux comprendre sans pour autant se laisser aller à dénigrer ses lectures antérieures. Mais à la deuxième relecture « on se défie un peu de soi » et on prend un peu plus ses distances avec le texte, ceci n'excluant pas le plaisir ni parfois le désir « d'inventer un peu à la suite de l'auteur. » On relit aussi «pour jouir du détail, du style. » L'attention se reporte alors sur l'écriture proprement dite du texte : «Nous entrons maintenant dans le laboratoire de l'auteur, nous le voyons travailler. » Enfin on relit pour se comparer à soi-même. On évalue ainsi le cheminement de sa sensibilité, de son intelligence critique. Et Emile Faguet conclut : «Relire, c'est revivre. »

Ces quelques remarques montrent combien nous sommes loin des relectures à l'identique évoquées dans l'introduction de cet article. Ces remarques permettent également de voir les enjeux d'un travail guidé de relecture dans la construction du lecteur. Et les activités proposées aux élèves ne sont pas, loin s'en faut, en rupture avec les quatre points dégagés par E. Faguet. Certes «se comparer à soimême » n'a pas été pris en compte (la longue durée n'a pas fait partie des paramètres retenus dans l'élaboration du projet). Par contre nous avons favorisé une posture complémentaire - « se comparer à des pairs »-qui est une façon de se confronter à d'autres semblables à moi et pourtant différents. Mais pour le reste, il s'agit bien de relire pour mieux comprendre et pour être plus attentif au travail de l'écriture c'est-à-dire passer d'une première lecture réactive à une deuxième lecture plus distanciée. Ou dit encore autrement, apprendre à pluraliser ses modalités d'appropriation des textes.

En effet dans un premier temps les élèves ont pratiqué une lecture à dominante éthico-pratique ${ }^{(14)}: 1$ 'adhésion à l'histoire et les réactions positives ou négatives sont sollicitées. C'est, par exemple, le rôle que jouent les post-it. L'identification au héros incite à faire jouer, sur un mode imaginaire, sa propre expérience de la vie : «passages à lire avec attention parce qu'on s'imagine vivre avec le héros » ou «pages que j'entoure quand j'aimerais ressembler au personnage ». Le lecteur, enfin, a été libre de refuser ce qui freinait sa participation, ce qui contrecarrait son désir d'être pris dans l'action («les passages que tu peux sauter»).

Dans un deuxième temps, les élèves sont outillés pour pratiquer une lecture à dominante esthético-formelle ${ }^{(15)}$ c'est-à-dire plus ancrée dans la réalité tex-

(13) E. Faguet, L'Art de lire, Armand Colin, 1992, pp. 139-145. Sur la relecture, voir aussi R. Barthes, $S / Z$, Editions du Seuil, coll. Points, 1970, pp. 22-23 ; E. Fraisse et B. Mouralis, Questions générales de littérature, Editions du Seuil, coll. Points, 2001, p. 216.

(14) Bernard Lahire, La Raison des plus faibles. Rapport au travail, écritures domestiques et lectures en milieux populaires, Presses universitaires de Lille, 1993, p. 103 et suivantes.

(15) Idem. 
tuelle et son appréciation littéraire. L'attention du lecteur porte plus directement sur la langue et les choix scripturaux réalisés, sur les types de textes, sur l'architecture du roman et les séquences récurrentes qui scandent l'avancée du récit, enfin sur la confrontation entre l'incipit et explicit.

Chaque groupe se retrouve donc dans une salle différente, accompagné par un enseignant ou le professeur documentaliste du collège. La deuxième partie de la fiche "Lire-Annoter-Relire » est alors exploitée : il s'agit de « relire pour mieux comprendre encore le livre et donc mieux le juger. » L'activité de relecture qui s'engage concerne plus particulièrement les pages choisies, celle dont le numéro a été entouré (séance 11).

Les élèves ont à travailler les trois points suivants :

1. L'écriture du texte. Sur une feuille cartonnée est recopié un extrait qui plait au lecteur (un dialogue, une description, un nom de personnage, des jeux avec les mots, des comparaisons poétiques, etc.)

Il ne s'agit pas ici de sacrifier à la mode du best-of et de la compil'mais bien de montrer, à travers la confrontation des points de vue, la diversité des préférences et donc la liberté de celui qui lit.

2. La construction du récit. Il est demandé de repérer un lieu, une action, une situation ou un problème qui se répète, qui est récurrent. Un exemple doit être donné.

3. Le message, le sens général de l'histoire. Les deux ou trois premières pages sont comparées aux trois dernières pour expliquer ce que l'auteur a voulu dire ou tout au moins ce que l'on a compris. Le lecteur doit aussi donner son avis sur le livre qu'il vient de lire. Pour justifier son interprétation, il cite au moins deux passages pris dans l'ensemble de la fiction car «les effets en retour permis par la relecture sont souvent indispensables pour apprécier, ou même simplement comprendre, tel passage textuel. » ${ }^{(16)}$

Ces trois activités explicitées, les élèves répondent sur trois fiches de couleurs différentes. Un rapporteur par fiche est désigné. Lors de la mise en commun (séance 12), chaque rapporteur présente les réflexions auxquelles son groupe est parvenu.

Ainsi le groupe du Bon Gros Géant cite ce dialogue unanimement qualifié d'humoristique pour illustrer l'écriture du texte :

"Avec la frambouille, dit Sophie, les bulles que vous avez dans le ventre se dirigent vers le bas et le résultat peut devenir encore plus désagréable.

Pourquoi désagréable? demande le Bon Gros géant en fronçant les sourcils.

Mais parce que, poursuivit Sophie en rougissant légèrement, si elles descendent au lieu de monter, elles sortiront par un endroit en faisant encore plus fort t plus malpoli. »

Le groupe du Sorcier aux loups a repéré dans la construction du récit des situations récurrentes (Saint-Roch est une sorte de sorcier qui a le don de parler aux animaux et le texte présente la scène du discours aux animaux plusieurs fois). Les lecteurs signalent également des lieux identiques (l'action se passe souvent dans la prison), des thèmes dominants (l'amour de Saint-Roch pour Adeline : première rencontre p. 44, il lui sauve la vie p. 78, les deux baisers p. 103 et p. 136, enfin l'amour récompensé dans les trois dernières pages du roman).

(16) Vincent Jouve, La Lecture, Hachette supérieur, coll. Concours Littéraires, 1993, p. 19. 
Pour le groupe de La Rédac, le sens général de l'histoire se résume ainsi : «L'auteur a voulu expliquer que les rédacs sont embêtantes et qu'il faut les faire à l'avance. » Pour justifier son interprétation, le rapporteur cite : «Et je me suis répondu en me tenant le bide, crispé par la souffrance, hérö̈que, à la Charles Bronson: - C'est rien, c'est vraiment rien, mon pote! Rien du tout! Laisse tomber! Juste une petite rédac à commencer. "

Quand les avis sont partagés, le rapporteur le signale. Les lecteurs de « La Rédac » ne sont pas parvenus à se mettre d' accord sur le choix des extraits à faire figurer dans la partie « écriture du texte». Deux citations étaient particulièrement en concurrence pour mettre en valeur la tonalité humoristique du récit. Elles ont été données toutes les deux. Le livre peut-être apprécié par certains, moins par d'autres, ces différences sont prises en compte. Il ne s'agit pas de gommer la diversité des lecteurs, mais bien de jouer de la pluralité des lectures et des stratégies mises en œuvre.

Il est procédé enfin au vote final. Chacun se détermine en fonction de ce qu'il a entendu et de ce qu'il pense. Un bulletin est distribué. Pour chaque rubrique (l'écriture du texte, la construction du récit, le sens de l'histoire), les trois romans qui concourent reçoivent une note qui va de 1 à 5 . Les deux livres qui obtiennent les meilleurs résultats sont les deux livres primés. Le Sorcier aux loups arrive largement en tête avec 271 points. Il est suivi par Le Bon Gros Géant qui obtient 210 points. Et reçoit donc le prix spécial. La Rédac avec 205 points est éliminée.

\section{Lire, un apprentissage (aussi) artisanal}

Dans la configuration livre - lecteur - lecture, le projet dont nous venons de rendre compte se place résolument du côté du lecteur. En effet, il ne s'agit ici ni de se centrer sur le choix des corpus de livres à lire, ni de privilégier le sujet cognitif et les analyses de textes, mais bien de valoriser le sujet social et culturel, et les processus d'appropriation qu'il construit (plus ou moins efficacement) pour mener à bien sa lecture.

Le bilan d'une telle activité peut se synthétiser de la façon suivante :

- diversifier les gestes de lecture en proposant aux lecteurs de confronter leurs pratiques avec celles des autres et d'en découvrir de nouvelles qu'ils peuvent éventuellement adopter ;

- faire intervenir la lecture et l'écriture car, tout au long du projet, les élèves lisent plusieurs livres mais écrivent aussi pour garder des traces de lecture, sorte de mémorisation écrite de leur pratique de lecteur ;

- offrir une situation active qui sollicite la coopération entre lecteurs en multipliant les échanges, les confrontations, les négociations ;

- familiariser les élèves avec des médiations, véritables micro-technologies de l'intellect littératien ${ }^{(17)}$ qui aident à la maîtrise pratique des textes écrits ; - responsabiliser les lecteurs en les impliquant dans l'interprétation et les jugements évaluatifs à porter sur les textes ;

(17) J. Goody, «La technologie de 1'intellect », La Littératie - Autour de Jack Goody, Pratiques, 131-132, décembre 2006, pp. 7-30. 
- enclencher une dynamique qui fasse travailler les représentations du travail de lecteur en substituant à la fatalité de la solitude et au primat exclusif du cognitif, l'entraide et l'élaboration de stratégies artisanales de lecteur.

Voilà un exemple de travail collectif que Jean-François Halté si attentif aux dispositifs pédagogiques, fussent-ils « artisanaux », aux objets d'enseignement liés à la praxis sociale et aux sujets culturels de l'apprentissage aurait peut-être accepté de considérer comme une tentative pour " apprendre autrement à l'école » ${ }^{(18)}$. C'est en tout cas ce que nous avons cru apprendre pour notre part en le lisant et en devisant si souvent avec lui.

\section{Bibliographie}

BARTheS, R. (1979) : Sollers écrivain, Seuil, p. 75.

Burgos, M. et PRIVAT, J.-M. : «Le Goncourt des lycéens : vers une sociabilité littéraire? ", Lire en France aujourd'hui, sous la direction de M. Poulain, Paris, Editions du Cercle de la Librairie, Collection Bibliothèques, 1993, pp. 163-181.

Goody, J. (2006) : «La technologie de l'intellect», La Littératie - Autour de Jack Goody, Pratiques, n¹31-132, pp. 7-30.

Halté, J.-F. (ss la dir. de) (1982) : «Travailler en projet», Pratiques, 1982, 36.

LAHIRE, B. (1993) : La raison des plus faibles. Rapport au travail, écritures domestiques et lectures en milieux populaires, Presses universitaires de Lille, p. 101 et suivantes.

Penloup, M.-C. (2000) : «Questions scolaires à l'écriture extrascolaire », Pratiques $\mathrm{n}^{\circ} 115-116$, pp. 1-9.

PRIVAT, J.-M. et VinSON, M.-C. (2000) : « Médiations culturelles et médiations textuelles au lycée », Pratiques, n¹07-108, Les nouveaux programmes au lycée, pp. 205-219.

PRIVAT, J.-M et al. (2001) : "Vers une didactisation des médiations textuelles », Cahiers du français contemporain, $n^{\circ} 7$, Pratiques de lecture et cheminements du sens, coordonnés par Michel Dabène, ENS éditions, Lyon, pp. 161177.

VInSON, M.-C. (2006) : «L'index, “une technologie de l'intellect" », Pratiques n¹31-132, La littératie. Autour de Jack Goody, pp. 199-216.

(18) J.-F. Halté, « Apprendre autrement à l'école », Travailler en projet, Pratiques, 36, 1982, pp. 5-22. 


\section{ANNEXES}

\section{FICHE $n^{\circ} 1$}

Lire 4 livres de littérature de jeunesse en cinq semaines et décerner après discussions le Grand Prix et le Prix Spécial « Serial Readers » de la classe de $6^{\mathrm{e}} 2$ du collège... en collaboration avec le CDI.

Nous avons commencé par constituer ensemble notre corpus de lectures : 30 livres du CDI (un livre par séries).

10 livres de fiction par groupe de 7 élèves pour en sélectionner 4 ( chaque groupe doit en éliminer 6).

Elaboration de critères de choix pertinents

Critère 1 : éliminer les livres trop longs

Critère 2 : éliminer les livres trop faciles ou trop difficiles

Critère 3 : éliminer les livres connus

Critère 4 : éliminer les livres déjà lus.

Les 10 livres qui ont été retenus en vue d'une prochaine sélection sont :

- A. Arkin, Moi, un lemming, Castor Poche, Flammarion.

- E. Brisou-Pelen, La Cour aux étoiles, Cascade, Rageot Editeur.

- N. Ciravegna, Chichois de la rue des Mauvestis, Bordas.

- R. Dahl, Le Bon Gros Géant, Folio junior, Gallimard.

- L. Garfield, Le fantôme de l'apothicaire, Folio junior, Gallimard.

- J. Mirande, Double meurtre à l'abbaye, Castor Poche, Flammarion.

- M.-A Murail, Baby-sitter blues, Médium poche, L'Ecole des loisirs.

- J.C Nogués, Le faucon déniché, Le livre de poche jeunesse, Editions GP.

- E. Reberg, La rédac, Arc-en-pohe, Nathan.

- P. Thiés, Le sorcier aux loups, Cascade Fantastique, Rageot Editeur.

\section{FICHE $n^{\circ} 2$ MES CONSEILS DE LECTEUR}

$1^{\circ}$ Pour t'aider à organiser ton temps de lecture :

- la durée moyenne de ta lecture

- les grandes étapes du texte à lire

$\mathbf{2}^{\circ}$ Pour t'aider à organiser ton parcours de lecteur :

- les passages que tu peux sauter (lis mes raccourcis)

- les passages que je te conseille de lire avec plus d'attention

$3^{\circ}$ Pour t'aider à vraiment bien comprendre le texte :

- en lisant ce passage, ce chapitre, ce livre tu peux penser à... 
- pour t'éclairer sur ce passage, ce chapitre, ce livre je te propose cette illustration....

- pour que tu avances plus vite que moi, je t'explique...

$4^{\circ}$ Pour te faire partager mes réactions de lecteur (tu peux en avoir d'autres !),

- tu rencontreras mes trois post-it « super»

- tu tomberas sur mes trois post-it « bof»

$\mathbf{5}^{\circ}$ Et surtout n'oublie pas que si tu es en difficulté, tu as intérêt à faire appel à des lecteurs plus experts (ton professeur, ta documentaliste, ton bibliothécaire, ton libraire, tes parents, d'autres élèves...)

Mais on peut aussi en parler ensemble.

Mon Nom :

\section{FICHE $n^{\circ} 3$ \\ NOS CONSEILS DE LECTEURS}

Mêmes items que pour la fiche 2 . La seule modification à apporter est la transformation du « je » en «nous » qui marque la coopération des lecteurs.

\section{FICHE ${ }^{\circ} 4$ \\ NOS NOUVEAUX CONSEILS DE LECTEURS}

$1^{\circ}$ Pour te faire partager nos réactions de lecteurs sur une page ou un court passage (tu peux avoir un autre avis !) :

- tu trouveras nos trois post-it « super»

- tu tomberas sur nos trois post-it « bof»

*** On te dit pourquoi en quelques mots sur chaque post-it.

$2^{\circ}$ Pour t'aider à organiser ton temps de lecture :

- la durée moyenne de la lecture

- les grands «blocs » de texte qu'il vaut mieux lire sans s'arrêter

$3^{\circ}$ Pour $\mathrm{t}$ 'aider à organiser ton parcours de lecteur :

- les passages que tu peux sauter (lis nos raccourcis)

- les passages que nous te conseillons de lire avec plus d'attention (amusants, bien écrits, importants pour comprendre, émouvants, pleins de suspense, surprenants, bien illustrés, on s'imagine vivre avec le héros, etc.)

$4^{\circ}$ Pour t'aider vraiment à comprendre le texte :

- la lecture du livre nous a fait penser à d'autres histoires (films, livres, BD)

- pour que tu avances plus vite que nous, nous t'expliquons 
* quelques mots importants que nous avons trouvés difficiles

* ce passage du livre qui n'est pas très clair à première lecture et sur lequel on a intérêt à réfléchir

$5^{\circ}$ Et surtout n'oublie pas que si tu es en difficulté, tu as intérêt à faire appel à des lecteurs plus experts (ton professeur, ta documentaliste, ton bibliothécaire, ton libraire, tes parents, d'autres élèves...)

Mais on peut aussi en parler ensemble.

De la part de (nos noms) :

\section{FICHE $n^{\circ} 5$ \\ COMMENT CHOISIR UN LIVRE ? NOS CRITERES}

1. Vocabulaire proche du lecteur

2. Texte compréhensible

3. Nombre de personnages limité

LISIBILITE

4. Importance des dialogues

5. Rapport au réel

6. Histoire contemporaine

7. Intérêt du thème dominant

8. Intérêt maintenu tout au long INTERET

9. Roman avec de l'action

10. Suspense

11. Humour

12. Découverte d'un auteur nouveau

13. Originalité du style (extrait)

NOUVEAUTE

14. « Morale » de l'histoire

15. Public visé

16. Collection

CLASSIFICATION

17. Sous-genre

18. Qualité du titre

19. Dédicace

20. Illustrations intéressantes

21. Dossier d'informations

22. Explications en $4^{\circ}$ de couverte

OBJET-LIVRE

23. Qualité de la présentation matérielle

24. Typographie

25. Nombre de pages 


\section{FICHE ${ }^{\circ} 6$ \\ LIRE-ANNOTER-RELIRE}

Nom du lecteur :

Titre du livre :

Nom de l'auteur:

1. Je réagis personnellement au fil de ma lecture aux actions et paroles des personnages, à l'imagination de l'auteur, aux passages trop difficiles ou trop "bébé».

Petit matériel: Un crayon à papier, une gomme.

Travail : J'entoure au crayon le numéro de la page

- quand j'aimerais ressembler au personnage

- quand je suis d'accord avec ce qu'il fait

- quand je le trouve amusant, mystérieux, intelligent, etc.

- quand je trouve que l'histoire est bien imaginée.

Je barre au crayon le numéro de la page :

- quand je n'aimerais pas ressembler au personnage

- quand je ne suis pas d'accord avec ce qu'il fait

- quand je ne le trouve pas intéressant

- quand je trouve que l'histoire est banale, ennuyeuse, etc.

- quand une page me paraît trop compliquée.

2. Je relis pour mieux comprendre le livre et donc mieux le juger.

Matériel: Trois fiches cartonnées.

Travail: Je reviens essentiellement sur les pages dont le numéro est entouré.

- L'écriture du texte (dialogues, descriptions, noms de personnages, jeux avec les mots, comparaisons poétiques, etc.) Je recopie sur une fiche cartonnée quelques extraits représentatifs qui me plaisent. Je n'oublie pas d'indiquer le numéro des pages.

- La construction du récit. Je repère un lieu, une situation ou un problème qui se répètent, que l'on retrouve plusieurs fois. Je donne un exemple et je dis si c'est bien. J'utilise la deuxième fiche cartonnée.

- Le message, le sens général de l'histoire. Je compare les deux ou trois premières pages du début avec celles de la fin du livre. J'explique ce que je comprends que l'auteur a voulu dire, montrer. Je donne mon avis par écrit. Sur cette troisième fiche, je donne des preuves de mon interprétation en citant au moins deux ou trois passages pris dans l'ensemble de la fiction. Je note le numéro des pages. 


\section{SYNOPSIS DES SÉANCES}

Séance 1 : Constitution du corpus.

Les élèves constituent un corpus de 10 livres en utilisant des critères (Fiche ${ }^{\circ} 1$ ).

Séance 2 : Lecture individuelle.

La classe est divisée en 5 groupes. Dans chaque groupe, 4 élèves reçoivent 2 livres différents (chaque livre est donné en double exemplaire). La fiche $\mathrm{n}^{\circ} 2$ est présentée par les enseignants. La lecture individuelle de premier livre commence. Elle se poursuit à la maison.

Séance $\mathbf{n}^{\circ} \mathbf{3}$ : Préparation de la lecture d’un pair.

Chaque élève finit de lire son livre et remplit la fiche $\mathrm{n}^{\circ} 2$.

Séance $n^{\circ} 4$ : Coopération entre lecteurs.

Dans chacun des 5 groupes, les deux élèves qui ont lu le même livre échangent leur fiche $n^{\circ} 2$. Ils discutent et remplissent ensemble la fiche $n^{\circ} 3$. Puis les livres accompagnés de leur fiche sont échangés à l'intérieur du groupe.

Séance $n^{\circ} 5$ : Lecture outillée.

Dans chacun des 5 groupes, les élèves lisent leur deuxième livre en utilisant la fiche $n^{\circ} 3$. La lecture se déroule en classe et à la maison.

Séance $\mathbf{n}^{\circ} \mathbf{6}$ : Critères de choix et première sélection de livres.

En classe entière, les élèves établissent une liste de critères utiles pour choisir un livre. Puis comme dans chaque groupe, les élèves ont lu les mêmes livres, ils désignent celui qu'ils préfèrent en se référant aux critères dégagés. Un livre par groupe est donc sélectionné.

Séance $\mathbf{n}^{\circ} 7$ : Coopération entre lecteurs.

Dans chaque groupe, les lecteurs remplissent ensemble la fiche $\mathrm{n}^{\circ} 4$ qui porte sur le livre sélectionné. Puis les livres sélectionnés sont échangés d'un groupe à l'autre. Les élèves se mettent à lire leur troisième livre. La lecture se poursuit à la maison.

Séance $\mathbf{n}^{\circ} \mathbf{8}$ : Critères de choix et deuxième sélection de livres.

Dans chacun des 5 groupes, les 4 élèves finissent de lire leur livre en utilisant la fiche $n^{\circ} 4$. Ensuite ils opèrent une nouvelle sélection : ils comparent le troisième livre qu'ils viennent de lire au deuxième qu'ils ont déjà lu. Pour construire leur choix, ils utilisent une liste de critères $\left(\right.$ Fiche $\left.n^{\circ} 5\right)$ : cette liste reprend les critères dégagés lors de la séance 6 mais cette fois ils sont classés et dotés d'une note. Les trois livres qui obtiennent le plus gros score restent en course.

Séance $\mathbf{n}^{\circ} 9$ : Préparation à la relecture.

La classe est répartie en 3 groupes de 7 élèves. Dans chaque groupe, les élèves lisent le même livre ( le quatrième livre). Ils entourent au crayon les numéros des pages qui les intéressent et barrent les autres ( $c f$. Fiche $\left.n^{\circ} 6\right)$. La lecture se finit à la maison. 
Séance $\mathbf{n}^{\circ} \mathbf{1 0}$ : Coopération entre lecteurs.

Dans chaque groupe, la mise en commun permet d'échanger sur les pages retenues, celles dont le numéro a été entouré.

Séance $n^{\circ} 11$ : Relecture.

Dans chaque groupe, les élèves relisent essentiellement les pages entourées et travaillent trois points $\left(c f\right.$. Fiche $\mathrm{n}^{\circ} 6,2^{\mathrm{e}}$ partie). Ils désignent un rapporteur par point travaillé.

Séance $\mathbf{n}^{\circ} 12$ : Jury de lecteurs.

Mise en commun devant la classe : les rapporteurs de chacun des trois groupes viennent, à tour de rôle, présenter les résultats de leur relecture. Puis il est procédé au vote final. 